\section{A case of scarred uterine rupture at 11 weeks of gestation having a uterine scar places induced by in vitro fertilization-embryo transfer}

\author{
Akiko Takashima, Naoki Takeshita, \\ Toshihiko Kinoshita \\ Department of Obstetrics and \\ Gynecology, Toho University Medical \\ Center, Sakura Hospital, Japan
}

\begin{abstract}
Having a uterine scar places a woman at increased risk of complications, such as Cesarean scar pregnancy (CSP), uterine rupture, placenta previa, and placenta accreta, in subsequent pregnancies. We report a case of uterine rupture at 11 weeks of gestation in a woman with a previous Cesarean section. A 43-year-old woman with a history of abdominal myomectomy and Cesarean section had her pregnancy induced by in vitro fertilization with donor eggs. The exact location of the gestational sac was identified on her first day of hospitalization, and her pregnancy was suspected to be a CSP. The following day, the patient complained of sudden lower abdominal pain. A uterine scar rupture was diagnosed, and an emergency surgery was required. It may be that first-trimester screening could allow the early recognition of patients at risk for these perinatal complications.
\end{abstract}

\section{Introduction}

Cesarean scar pregnancy (CSP) is rare but may lead to life-threatening complications. The increasing rate of Cesarean delivery plus the rapid development of in vitro fertilization-embryo transfer (IVF-ET) technology may increase the occurrence of CSP. Uterine rupture, defined as disruption or tear of the myometrium and serosa of the uterus, is a life-threatening condition for both the mother and her fetus. It may be seen in the second trimester, usually after induction for pregnancy termination in a scarred uterus. A spontaneous uterine rupture in the first trimester is a very rare event. Here, we report a case of scarred uterine rupture at 11 weeks of gestation induced by IVF with donor eggs.

\section{Case Report}

This patient was a 43-year-old female who married 5 years ago. She had a history of infertility for 4 years. She had undergone abdominal myomectomy at age 35 , during which 6 myomas were excised from her uterus, and a Cesarean section (CS) was performed at age 42. Her first pregnancy was induced by IVF with donor eggs. Placenta previa was diagnosed by sonographic examination at 23 weeks of gestation. The patient was advised to terminate her pregnancy by Cesarean section at 25 weeks of gestation because of uncontrollable genital bleeding. After evacuating the baby and placenta, a transverse fundal incision was repaired with two suture layers. The patient had no history of abnormal bleeding after CS. The present pregnancy was also induced by IVF with donor eggs. There was no mention of her uterine scar before embryo transfer at the 10th week of gestation (Figure 1). The exact location of her gestational sac (GS) was determined at the first day of hospitalization at 11 weeks of gestation; her GS had implanted in the scar or the niche of a previous Cesarean delivery (Figure 2). The diagnosis was based on finding a GS at the site of the Cesarean scar in the uterine cavity associated with a thin myometrium by transvaginal ultrasound. We suspected that the pregnancy was a CSP. After careful examination, radiological imaging and management options were planned. The following day, the patient complained of sudden lower abdominal pain. Observations showed a pulse of 106 beats per min and a blood pressure of $84 / 46 \mathrm{mmHg}$. Emergent sonography in the emergency ward showed free fluid in the cul-de-sac and pelvic fossa and no fetal heartbeat in the GS found in the abdominal cavity, near the abdominal wall. Uterine rupture was diagnosed. An emergent laparotomy was performed. Intraoperative findings revealed approximately $1300 \mathrm{~mL}$ blood in the abdominal cavity and between the intestinal loops. The uterus was torn at the fundus, and all gestational sac contents with the fetus had moved away from the tearing zone. Rupture was identified in the uterine fundus and cornea of the Cesarean scar (Figure 3). Hysterectomy was performed, and 12 units of packed red blood cells and 8 units of frozen fresh plasma were transfused. The patient was later discharged from the hospital in good condition.

\section{Discussion}

Uterine rupture during pregnancy may
Correspondence: Akiko Takashima, Department of Obstetrics and Gynecology, Toho University Medical Center, Sakura Hospital, 564-1 Shinoshizu, Sakura, Chiba 285-0841, Japan.

Tel.: +81.043.462.8811 - Fax: +81.043 .489 .5353$ E-mail: takashima-04@sakura.med.toho-u.ac.jp

Key words: Cesarean scar pregnancy; uterine rupture; in vitro fertilization-embryo transfer.

Contributions: Dr. Takashima drafted the manuscript, with the help of Drs. Takeshita and Kinoshita. All the authors read and approved the final manuscript.

Conflicts of interest: the authors declare no conflict of interest.

Received for publication: 5 November 2017. Accepted for publication: 3 April 2018.

This work is licensed under a Creative Commons Attribution NonCommercial 4.0 License (CC BY-NC 4.0).

(C) Copyright A. Takashima et al., 2018

Licensee PAGEPress, Italy

Clinics and Practice 2018; 8:1038

doi:10.4081/cp.2018.1038

occur in the entire uterine wall thickness and uterine serosa. Different factors increase this risk such as congenital uterine anomalies, multiparity, previous myomectomy, labor induction, uterine trauma and previous uterine scars, including Cesarean section, abdominal and laparoscopic surgeries, and dilation and curettage (D\&C). The rate of total uterine rupture has been reported to be approximately $0.07 \%$. By comparison, the incidence of uterine rupture is $0.012 \%$ in cases without previous scars. Almost all cases of uterine rupture occur during the third trimester of pregnancy. ${ }^{1-3}$ Only 2 cases of spontaneous uterine rupture have been reported during the second trimester. ${ }^{4,5}$

CSP is defined as an ectopic pregnancy (EP) implanted in the myometrium of a Cesarean section scar. ${ }^{6}$ The incidence of CSP is extremely low and has been estimated to range between $1 / 1800$ and $1 / 2500$ of all Cesarean deliveries performed. The predisposing risk factors include uterine trauma, Cesarean section, abdominal and laparoscopic surgeries, D\&C, IVF-ET and manual removal of placenta. $^{7}$ IVF-ET could be a sole risk factor even without any previous uterine surgery. ${ }^{8}$ Although a uterine scar is a well-known risk factor for CSP or uterine rupture, the sonography plays a very important role in precisely locating the GS.

The first trimester of pregnancy is increasingly considered as a starting point 


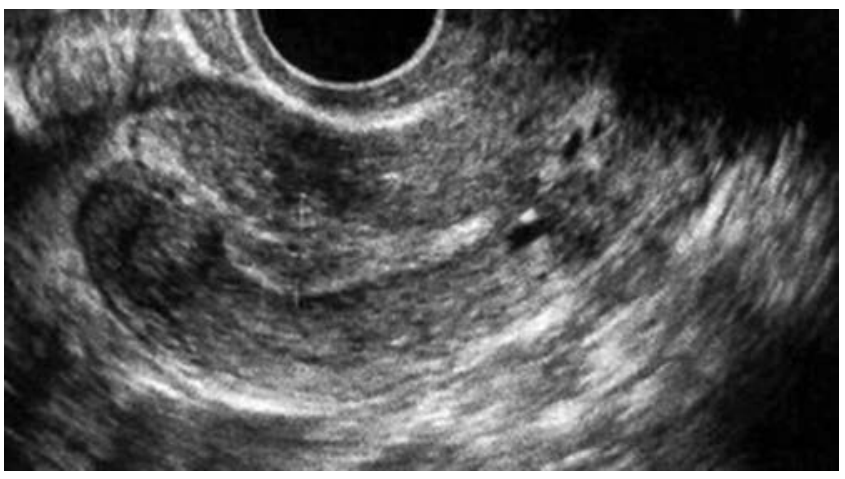

Figure 1. Sonography before embryo transfer.

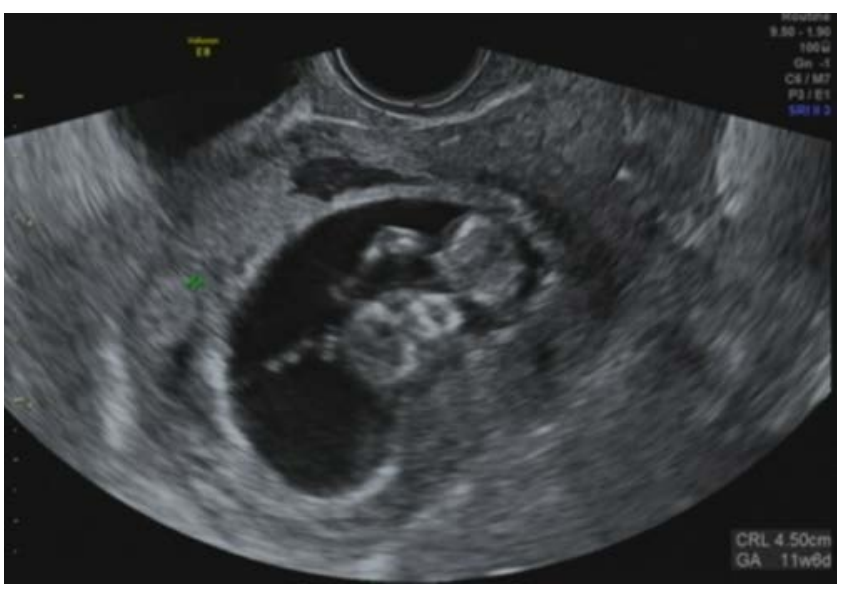

Figure 2. Exact location of the gestationl sac with the fetus.

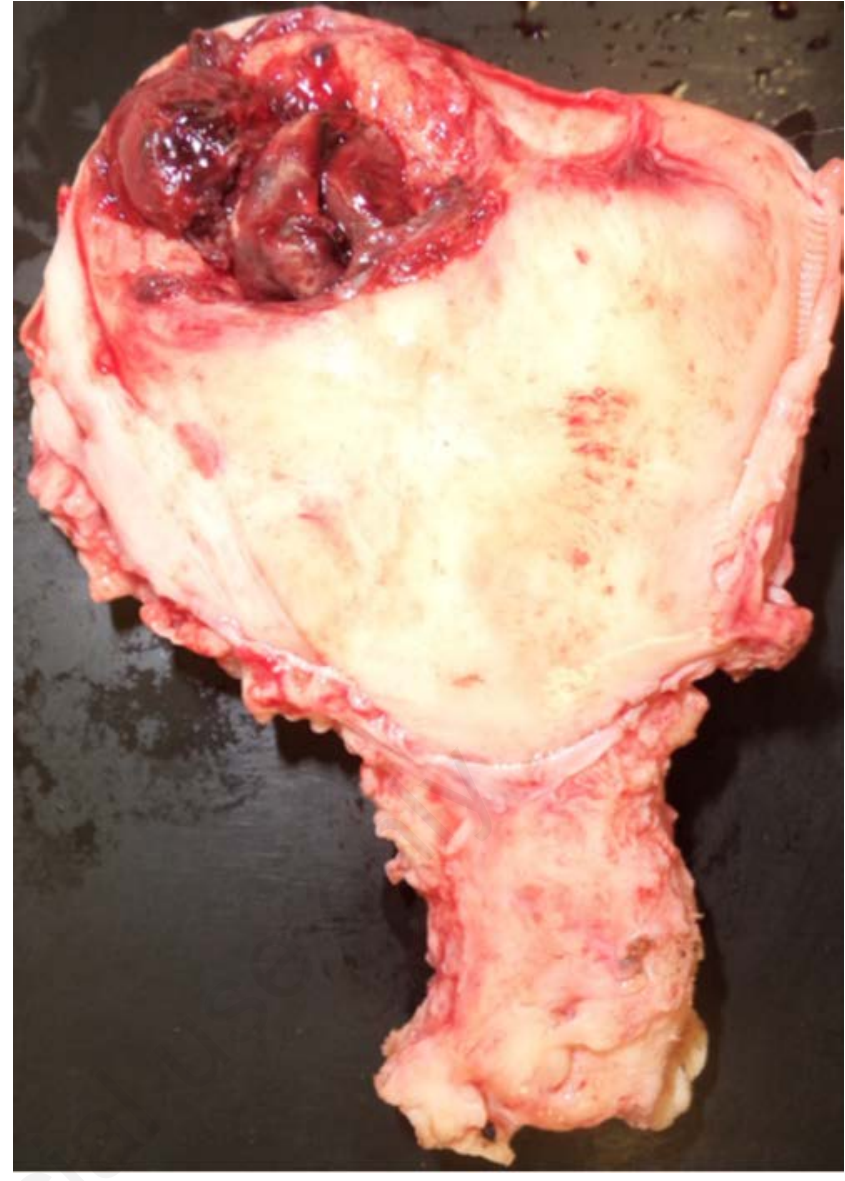

Figure 3. Uterine rupture at the previous Cesarean scar. for stratifying patients at risk of uterine rupture. Most Cesarean sections were carried out using a transverse lower segment incision. As the non-sonographic symptoms of transverse fundal incision are unclear, this case of CSP went undetected initially. The diagnosis of suspected CSP has been reported between 5 and 8 weeks of gestation. Most cases of CSP have been diagnosed in the first trimester by transvaginal ultrasound. ${ }^{9-11}$ In this patient, uterine rupture at 11 weeks of gestation is a rare event.

The optimal treatment of the patient in the first trimester of pregnancy with a sonographic diagnosis of suspected CSP remains uncertain. The proposed treatment involves one main treatment alone or its combination with other treatment modalities such as curettage, systemic MTX, hysteroscopy, laparotomy, and uterine artery embolization. ${ }^{10,11}$ Because our patient was bleeding acutely due to a uterine rupture, emergent surgery was required.

As the number of Cesarean births and the rapid development of IVF-ET have risen dramatically, so has the number of patients presenting for obstetric care with a previous uterine incision. This finding probably reflects the increasing proportion of obstetric complications, such as CSP or uterine rupture. The present case had multiple risk factors for uterine rupture, including Cesarean section, abdominal myomectomy and IVF-ET.

The first trimester of pregnancy is increasingly considered as a starting point for stratifying patients at risk of uterine rupture. It may be that first-trimester screening could allow the early recognition of patients at risk for these perinatal complications.

\section{Conclusions}

In a patient with a history of uterine surgery presenting with abdominal pain, uterine rupture must be suspected, even early in pregnancy. The first trimester is increasingly considered as a starting point for stratifying patients at risk of CSP or uterine rupture because of a history of lower-segment cesarean section. It may be that firsttrimester screening could allow the early recognition of patients at risk for any of these perinatal complications.

\section{References}

1. Gommers JSM, Diederen M, Wilkinson $\mathrm{C}$, et al. Risk of maternal, fetal and neonatal complications associated with the use of the transcervical balloon catheter in induction of labour: A systematic review. Eur J Obstet Gynecol Reprod Biol 2017;218:73-84.

2. Gardeil F, Daly S, Turner MJ. Uterine rupture in pregnancy reviewed. Eur J Obstet Gynecol Biol 1994;56:107-10.

3. Gambacorti-Passerini Z, Gimovsky A, Locatelli A, Berghella V. Trial of labor after myomectomy and uterine rupture: a systematic review. Acta Obstet Gynecol Scand 95:724-34.

4. Al-Ramahi M, Radi F, Qatawneh A, AlKazaleh F. Spontaneous uterine rupture at 16-week gestation after abdominal myomectomy. J Med J 2009; 43:351-4.

5. Golan D, Aharoni A, Gonen R, et al. Early spontaneous rupture of the post myomectomy gravid uterus. Int J Gynaecol Obstet 1990;31:167-70.

6. Lai YM, Lee JD, Lee CL, et al. An 
ectopic pregnancy embedded in the myometrium of a previous cesarean section scar. Acta Obstet Gynecol Scand 1995;74:573-6.

7. Rajakumar C, Agarwal S, Khalil H, et al. Caesarean scar pregnancy. J Obstet Gynaecol Can 2015;37:199-200.

8. Hamilton CJ, Legarth J, Jaroudi KA. Intramural pregnancy after in vitrofertilization and embryo transfer. Fertil
Steril 1992;57:215-7.

9. D'Antonio F, Timor-Trisch IE, Palacios-Jaraquemada J, et al. First trimester detection of abnormally invasive placenta in women at risk: a systematic review and meta-analysis. Ultrasound Obstet Gynecol 2017 [Epub ahead of print].

10. Timor-Tritsch IE, Monteagudo A. Unforeseen consequences of the increasing rate of cesarean deliveries: early placenta accreta and cesarean section scar pregnancy; a review. Am J Obstet Gynecol 2012;210:371-4.

11. Boza A, Boza B, Api M. cesarean scar pregnancy managed with conservative treatment. Iran J Med Sci 2016;41:4505. 